\title{
Towards Mobile Ad-Hoc WANs: Terminodes
}

\author{
J.-P. Hubaux, J.-Y. Le Boudec, M. Vojnović, S. Giordano, \\ M. Hamdi, L. Blazević, and L. Buttyán \\ EPFL-ICA*
}

February 2000

\begin{abstract}
Terminodes are personal devices that provide functionality of both the terminals and the nodes of the network. A network of terminodes is an autonomous, fully self-organized, wireless network, independent of any infrastructure. It must be able to scale up to millions of units, without any fixed backbone or server. In this paper we present the main challenges and discuss the main technical directions.
\end{abstract}

\section{Introduction}

The Terminode Project is a 10-year-long research program (2000-2010) [TN] that investigates wide area, large, entirely wireless networks that we call mobile ad-hoc wide area networks. In this project, we follow a radically distributed approach in which all networking functions are embedded in the terminals themselves [Hub99]. Because they act as nodes and terminals at the same time, we call these devices terminodes. A network of terminodes is an autonomous, self-organized network, completely independent of any infrastructure or other equipment. A previous paper presented the first technical options of terminodes [HLGH99].

Our vision of the Terminode Project can be illustrated by a scenario of a free, wireless network covering a wide area. In this scenario, terminodes are small personal devices owned by everyone in a given area (city, region or country). The set of terminodes constitutes a large network where multi-hop wireless communications allow voice and data messaging among all users. The whole network operates at unlicensed frequencies. It can be considered a free amateur wide area wireless network. The terminode users can be human or equipment, depending on the application. A terminode network can be of any size. In particular, in regions of high-density population, the size could reach several million devices. In the following, we summarize the main design points of the project.

The Spectrum is the Infrastructure: To eliminate the need for any additional device or network equipment, all networking functions (typically performed in backbone routers/switches and servers) are distributed in the terminodes. The only external resource needed by users is the frequency bandwidth that is assumed to be allocated by regulation authorities. The fact that routing/switching functions are performed in the terminodes dramatically changes the

${ }^{*}$ This is technical report DSC/2000/006, Communication Systems Department, Ecole Polytechnique Federale de Lausanne (EPFL), CH-1015 Lausanne, Switzerland. The authors are with the Institute for computer Communications and Applications (ICA), EPFL. Email: \{Jean-Pierre.Hubaux, Jean-Yves.LeBoudec, Milan.Vojnovic, Silvia.Giordano, Maher.Hamdi, Ljubica.Blazevic, Levente.Buttyan $\}$ @epfl.ch 
routing paradigm. A backbone of routers or switches typically looks like a tree, sometimes augmented with few redundant links. In the terminodes approach, the backbone is identical to the set of terminodes and looks more like a strongly connected graph with a very high level of redundancy.

Scalability to Large Numbers: Scalability to a very large number of terminodes is central to our research. In the Internet or Telecom networks, this issue is efficiently addressed using centralized and/or hierarchically organized routers and servers. This approach is inappropriate in our context.

Decentralization and Self-organization: Terminodes are designed to be self-organizing: any number of terminodes that form a connected graph can constitute a network. Therefore, all terminodes have a common, minimal set of functions that are necessary and sufficient for the network self-operation (peer-to-peer [Ver98]). Compared to current networks, the mechanisms that include centralized storage or processing must be substituted with completely distributed solutions. However, this does not imply that all terminodes are identical. A terminode can be individually extended with large processing, storage or internetworking capabilities that could be a benefit for entire community of terminodes - under the condition that these extensions are not necessary to run the network.

CB Business Model: The terminodes introduce an original business scenario in multimedia communication services. In today's networks, most multimedia communication services, including those supported by the Internet, are seen by the end user as commercial services that include a service contract and regular fees. In the scenario we consider, the paradigm is radically different: terminodes are goods that people purchase once and use forever, without service contracts or per-use-basis fees. This is similar to the business model of citizen band, amateur radio, and talkie-walkie systems.

\section{Related Work}

Research in mobile ad-hoc networks was initiated in DARPA Packet Radio projects [JT87]. The research on wireless networks has been mainly focused on cellular systems that are, in principle, single-hop wireless systems. Within the framework of the multi-hop wireless systems, research communities worked on projects that addressed mainly Medium Access Control (MAC) and routing issues.

The MAC layer specified in the IEEE 802.11 standard [802.11], or its variants, is typically assumed in the existing ad-hoc network projects. The standard is based on the Carrier Sense Multiple Access with Collision Avoidance (CSMA/CA) scheme that is extended with short channel allocation and acknowledgment control messages. Apart from IEEE 802.11, there have been many extensions to the basic CSMA/CA protocol, including MACA, MACAW, FAMA, and CARMA. Their common objective is to resolve contention to a single-channel for a limited number of transceivers. ${ }^{1}$ To probe further on these protocols, the reader is referred to [GLAF99] and the references therein. The wireless LAN technology is already widely available commercially. More recently, the Bluetooth standard has been defined [Blu99] as a low cost pico-cell wireless technology suitable for purposes such as cable replacement. However, it is still unclear how profitable it can be for mobile ad-hoc networks. Nevertheless, none of these protocols are targeted to deploy a wide-area mobile ad-hoc network such as the Terminodes network.

\footnotetext{
${ }^{1}$ An exception is CARMA, which has been extended to a multi-channel setting.
} 
In the last few years, projects such as Monarch, Wins, etc., produced several routing protocols, simulation tools, simulation analysis, and performed trials. Mobile ad-hoc networks are becoming accepted as a valid commercial concept, which is confirmed by the creation of the MANET working group in IETF [MAN]. Past work has focused on small networks. Based on the existing routing protocols, significant effort is currently being made in order to achieve scalability [ICP ${ }^{+}$99],[MZ99],[MBJJ99],[HP99]. Rather than following an evolutionary approach, we decided to investigate a different type of routing based on the physical location of the destination. A similar idea is used in [KV98], [BCSW99], [BCS99], but we combine it with new location and packet forwarding algorithms, designed specifically for networks with several million nodes.

\section{Basic Mechanisms}

In this section we outline the fundamental mechanisms that we envision. The following sections will address the mobility management, security and incentive to collaborate. Finally, we will sketch some application scenarios.

\subsection{Packet Switching and Burnt-In Addresses}

We envision a terminode network based on packet switching. Although circuit switching is an advantage for supporting voice (very small delay in relaying terminodes), the complexity associated with establishing, maintaining, and releasing circuits, or any form of connection, is at odds with the requirement that intermediate systems are user equipment, and may operate quite irregularly. Thus, we use connection-less packet switching. Delay will be minimized by supporting cut-through operations: routing information is placed at the beginning of the packet header and forwarding starts as soon as valid routing information has been analyzed.

Terminodes must be identified by some means of addressing. We need an address that a terminode can use without configuration; every terminode has a burnt-in 64 bit End-system Unique Identifier (EUI), as is planned today in the replacement of MAC addresses for any communication equipment. Note that terminodes are able to work with the Internet protocol (see below), however it is not a good idea to use IP addresses as the unique identifiers because, as long as IPv6 is not really deployed, there are not enough IPv4 addresses that could be set-aside for the exclusive use of terminodes.

\subsection{Radio System Architecture}

The concept to design a wide-area radio network with no fixed infrastructure raises several technical issues, which have not been of concern with the existing systems (e.g. cellular systems and wireless LANs). In this section we discuss the radio system architecture, including physical and MAC layers. Currently, the predominant radio access technology considered for the third-generation cellular systems is Code Division Multiple Access (CDMA) [OP98], [UMT97]. This is mainly due to an intrinsic flexibility of CDMA systems in terms of the radio resource planning. We envision the terminodes to use one, or several, non-operated frequency bands to be allocated for that purpose.

How should the radio system be architectured for terminodes? This question is raised bearing in mind that the system has to be decentralized and self-organizing, that it should cover a wide-area, support a potentially enormous number of terminodes, with a high diversity 
of terminode density. Architecturing the radio technology is a part of our ongoing research efforts, and our current considerations are centered around CDMA. Below, we address some of the technical issues involved.

We consider two tentative (global) radio system architectures that do not exclude other potential approaches.

- Terminodes are self-organized into communities. A community would be defined as a set of terminodes accessible by at least one of them in a single hop. This concept is fairly similar to the existing systems (cells of the cellular systems or individual wireless LANs). The basic distinction is that the communities are not assumed to be fixed to a given geographical region, and there is no notion of a base-station. The radio resource allocation could be done differently in intra- and inter-community communication. This is also inline with the packet forwarding discussed in section 3.3.

- A terminode is not a member of any specific community. In this approach, there is in fact no defined community. This concept is inspired by Shepard's paper [She96], which proposes an architecture based on CDMA. The author conducts a feasibility study to operate a dense packet radio network, where most of the radio resource allocation is resolved through CDMA, and only a simultaneous transmission and reception of a given station is alleviated through the MAC protocol. It is shown that the system is feasible assuming that the minimum energy routes are used, where each station directly communicates only with a few of its closest neighbors. However, it is demonstrated that localization of the traffic is necessary to avoid congestion, and this remains to be resolved. The paper, also, does not cover the mobility and code assignment problem. The latter may be needed to avoid the hidden-terminal problem [TK75], and can be solved through either transmitter, receiver, or pair-wise oriented code assignment [Hu93].

Indeed, one could argue whether the former or latter concept should be favored, which goes beyond the scope of the present paper. In general, common to all CDMA systems is the necessity for the transmission power control to reduce interference and to achieve a certain level of QoS (typically expressed in terms of a signal-to-interference ratio). Although, there is substantial work on the power control, most of the results are derived for the cellular systems setting. Hence, this is a potential direction of future work. Nevertheless, advanced concepts have to be evaluated to a full extent in order to define an architecture viable in the long-term (e.g. multi-user detection, rate adaptation, adaptive antennas).

Another issue of interest for the Terminodes is reduction of the power consumption in order to extend the battery longevity for battery supplied terminodes. There has been some work on designing power-aware protocols. For a recent overview of this issue the reader is referred to [WESW98].

\subsection{Packet Forwarding}

Since packet relaying is performed by terminodes themselves, the intermediate system functions should be as simple as possible, as is the case, for example, with source routing bridges. In a large, self-operated network, it may not be feasible to use routing protocols that distribute in one way or another some knowledge about the topology of the network. In contrast, we propose to use a combination of two types of packet forwarding, as described below. To this end, every terminode has, in addition to its permanent EUI, a temporary, location-dependent 
address (LDA). The LDA is simply a triplet of geographic coordinates (longitude, latitude, altitude), obtained for example by means of the geographic positioning system (GPS) ${ }^{2}$.

A terminode network can be presented, in a macroscopic way, as being composed of a number of blocks populated by terminodes. Terminodes organize themselves in blocks and each block can be seen as a group of terminodes that know how to communicate to each other. We assume that the spatial distribution of terminodes in each block follows the process that is known (e.g. Poisson or Markov Field). In addition, given the communication model inside the block, traffic patterns can be estimated through every block. Depending on the density of terminodes, traffic patterns in a block, and some additional information, we can characterize transit capability of a block. Thus, the macroscopic presentation of a block is given by its transit capability (capacity). Every terminode has a macroscopic view of all blocks and their transit capabilities.

Routing in a highly dynamic network without a fixed infrastructure should be different from the traditional routing. Traditionally, the shortest path routing is applied in the global Internet, which has a characteristic hierarchical topology. At the higher level is the backbone. In this case, most of the packets in the network are routed via the backbone links. When the traffic demands are increased then the backbone links become saturated. Since the backbone links are fixed, network management can easily respond to expansion of the network. In this case it should be sufficient to uniformly upgrade the backbone links in order to manage increasing traffic demands.

Within a network composed of terminodes, network topology is highly dynamic and unpredictable (there is not a fixed backbone). In addition, it is not possible to increase the capacity of links as a response to increasing traffic demands. Therefore, dynamic routing is performed with an objective to maximize the global utility, under the constraints imposed by the transit capabilities of the blocks that constitute a terminode network. We define the global utility $U(\cdot)$ as the sum over the user utilities minus the cost imposed by the network [Kel99]

$$
U(x)=\sum_{s \in \mathcal{S}} U_{s}\left(x_{s}\right)-\sum_{b \in \mathcal{B}} C_{b}\left(f_{b}\right),
$$

where $x=\left(x_{s}, s \in \mathcal{S}\right)$, and $\mathcal{S}, \mathcal{B}$ are defined to be sets of the sources and blocks, respectively. Source $s \in \mathcal{S}$ is characterized by a utility function $U_{s}(\cdot)$ that is assumed to be a concave increasing in its transmission rate $x_{s}$. For instance, one may consider sources to be controlled by a TCP-like rate control algorithm, and define $U_{s}(\cdot)$ appropriately. Total cost of the network, the right-most term in (1), is given by the sum of the costs of each block $C_{b}$. The latter, $C_{b}$, is a function of the amount of traffic $f_{b}$ that traverses the given block $b$.

Figure 1 shows an example of a terminode network. The constituting blocks of the network are shown. The blocks colored in black are those that cannot be used for packet relaying (e.g. obstacles or blocks without terminodes). This figure also illustrates packet forwarding from terminode $S$ to terminode $D$. We distinguish packet forwarding inside a block and packet forwarding among the blocks.

For intra-block packet forwarding, terminodes inside the same block know the path to each other. In Figure 1, the intra-block packet forwarding is denoted with solid lines that

\footnotetext{
${ }^{2}$ Coding longitude and latitude with an accuracy of $10^{-4}$ grades gives a position accuracy of about 10 meters. With this accuracy, the total number of position triples is to the order of $10^{16}$, thus an LDA could be coded with 48 bits, approximately.
} 


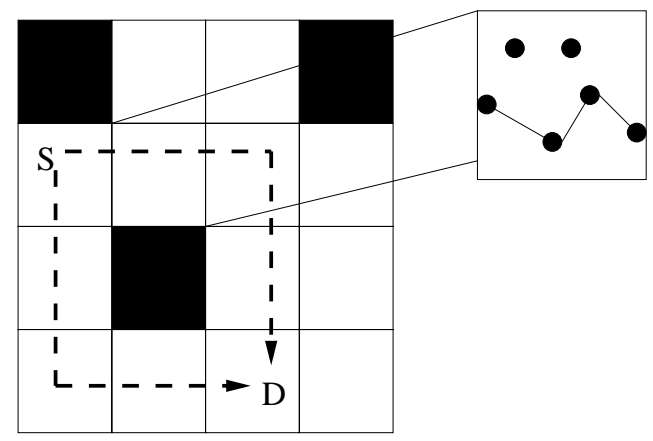

Figure 1: An example of a terminode network and its constituting blocks are shown. The blocks colored in black are those that can not be used for relaying packets. The figure also illustrates intra- and inter-block packet forwarding from terminode $S$ to terminode $D$.

represent connectivity among the terminodes inside the same block. Inside a block, one of the MANET [MAN] routing protocol proposals could be used.

For inter-block packet forwarding we propose the packet forwarding heuristic in order to optimize the global utility that is presented above. In this heuristic we use the knowledge of the geographical position of the destination (LDA), and of the block in which the destination resides (geodesic packet forwarding). In Figure 1, the inter-block packet forwarding is illustrated with dashed lines. The source may split its rate into several flows that are routed through differing paths to the destination.

Looping packets are discarded by the beans mechanism described below (similar to the TTL field in IP packets). Multicast packet forwarding in the Internet is already difficult enough and, thus for terminodes will be studied later.

\subsection{Terminodes and TCP/IP}

We will request a reservation of a portion of the IPv6 addressing space for terminodes; the IPv6 address of a terminode is then algorithmically mapped from its EUI. From an IPv6 viewpoint, the set of terminodes is one enormous subnetwork; IP sees the terminodes as one huge physical network. Two terminodes typically use the TCP/IP protocol stack to communicate. However, inside the network of terminodes, packet forwarding does not utilize IP addresses (see above), just as bridges operate in a large bridged network. Host routes may be configured in terminodes in order to force a packet that is destined to another terminode to reach a gateway connected to the rest of the Internet. Solutions that work with IPv4 are for further study. 


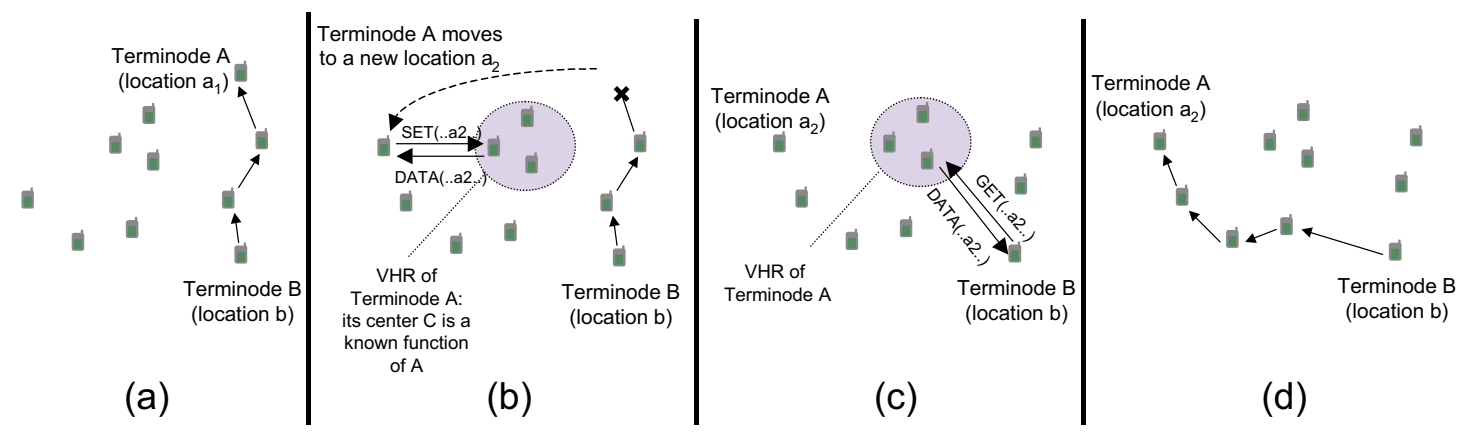

Figure 2: The VHR mobility management; (a) terminodes $A$ and $B$ are communicating directly, (b) terminode $A$ moves and uses the VHR protocol to publish its new position, (c) if $B$ does not directly keep track of $A$ 's movement, it uses the VHR protocol to retrieve the new location of $A$, (d) finally, $B$ re-establishes communication with $A$.

\section{Mobility Management}

In a network of terminodes, two generally adopted solutions to manage mobile terminals (flooding within MANET networks [MAN], and fixed server within GSM or Mobile IP) are not adequate and a new approach has to be found for mobility management. This is because the flooding operations have a dramatic effect in a very large network. In addition, no fixed infrastructure or servers can be assumed in the Terminodes network for the reasons mentioned above.

We have developed a new and original approach for mobility management that avoids both flooding, and servers or directory services. It is called the Virtual Home Region (VHR) approach.

\subsection{Principle}

A virtual home region is a set of terminodes located near to each other. The VHR is in charge of keeping some of the information generated by a terminode. This terminode is called the owner terminode (because it owns the information stored in the VHR). The VHR is defined by a point in the space, $C$, and a radius $R$. The terminodes that belong to a VHR are those located in the disk $(C, R)$. Each terminode defines a single VHR that we refer to as "its VHR"; the relation between a terminode and its VHR is defined by a well-known hash function $H$. The function $H$ operates on the EUI space and gives images in the LDA space: $H(E U I)=C$ with $C$ being the center of the VHR of terminode EUI.

When the owner terminode $A$ moves to a new location, it stores its new LDA coordinates in its VHR, using a simple SNMP-like protocol ${ }^{3}$. If a terminode $B$ is willing to send packets to $A$, it sends a query to $A$ 's VHR and retrieves its LDA. The VHR associated to $A$ is known to $B$ because $H$ is known to all terminodes (see Figure 2).

To make this approach work, terminodes are provided with the capability of storing short information, serving as temporary distributed memory for other terminodes. This cooperation is mandatory for a self-operating network.

\footnotetext{
${ }^{3}$ We also consider additional protocols for direct source-destination mobility tracking that are not discussed in this work.
} 
The advantage of this approach is that it requires a reasonable amount of communication to find a new position, in addition to the fact that no external devices or servers are necessary. There are several technical issues that are being solved to implement this solution; in particular the radius setting is discussed hereafter.

\subsection{The Radius Setting}

The VHR needs management procedures in order to ensure that it contains a sufficient number of terminodes. This number is appropriately chosen to ensure enough redundancy of the stored information and at the same time to be small enough to minimize communications inside the VHR. The expected VHR size is between 4 and 20 terminodes, mainly depending on the packet loss probability. The radius has then to be dynamically set to reach this size. Currently, two schemes are being evaluated to estimate the VHR size. In the first, the VHR is managed in a centralized mode (by the owner); in the second, it is managed in a distributed way (by the terminodes inside the VHR with self-organization functions). If the VHR is managed by its owner, it is the responsibility of the owner itself to estimate, monitor and manage the radius of its VHR. On the contrary, if the VHR is self-organized, this becomes a task of the terminodes in the VHR. The radius is increased when the VHR size falls under its minimum value and decreases when it exceeds its maximum value. A VHR Protocol is used to store and retrieve location information from the VHR, as illustrated in Figure 2.

\section{Security}

Security in networks (including wireless ad-hoc networks) is concerned with confidentiality and integrity of information, as well as legitimate use and availability of services [For94]. In military applications, confidentiality is considered to be the most important security objective. In civilian scenarios, however, availability has the greatest relevance for the user [SA99]. In the Terminode network, availability has two aspects:

- Stimulation for co-operation. Since all services (e.g. packet forwarding, mobility management) are provided by the terminodes themselves, these services are available only if the terminodes (or, more precisely, their users) are willing to provide them. On the other hand, service provision is not in the direct interest of users, because it consumes energy and, thus, reduces battery lifetime. Therefore, a stimulation mechanism is required that encourages users to leave their terminodes switched on and let them provide services to other terminodes. We discuss this issue further in Section 6, where we present an approach to solve this problem.

- Defense against denial-of-service attacks. Stimulation for co-operation is not enough to achieve availability, because services may be unavailable due to denial-of-service attacks, such as interception of packets and destruction or modification of control information (e.g. information required for the geodesic packet forwarding mechanism). Denial-ofservice attacks are typically impossible to prevent. However, they can be made very expensive by exploiting the inherent redundancy of the ad-hoc networks [ZH99]. For instance, a packet can be sent to its destination via several disjoint routes, which makes its interception considerably more expensive for the attacker. 
A fundamental tool to achieve network security objectives is cryptography. Cryptography is indispensable to confidentiality and to protection of the information integrity, and also used in mechanisms that ensure legitimate use of services (e.g. in authentication protocols). The challenge of using cryptography in the Terminode network is the management of cryptographic keys.

Since terminodes are mobile, their interactions are spontaneous and unpredictable, which makes public key cryptography more appropriate in this setting than conventional cryptography. The most widely accepted solution for the public key management problem is based on public key certificates that are issued by (off-line) certification authorities and distributed via (on-line) key distribution servers. Unfortunately, the application of certification authorities and key distribution servers contradict the self-organized ${ }^{4}$ and self-operated features of the Terminode network.

One approach for solving the key management problem may be based on the replacement of the certification authorities with communities of users (a PGP-like solution [Zim95]) and the distribution of the key distribution server function among the terminodes (a VHR-like solution). Another approach may be to adopt a system that implicitly guarantees the authenticity of public keys, such as identity-based systems [Sha85] and those using implicitly certified keys [Gir91].

\section{Incentive to collaborate}

As we mentioned in Section 5, the Terminodes network relies on the co-operative behavior of the terminodes (or, more precisely, their users). One possible approach to stimulate such behavior is to introduce the concept of money and service charges. The natural idea is that terminodes that used a service should be charged and terminodes that provided a service should be remunerated.

We call the Terminode currency beans. Beans can be created by international treaty organizations, and have no monetary value. We assume that the terminode hardware comes with an initial stock of beans.

One of the main services that the terminodes should provide to each other is the packet forwarding. We are investigating two approaches for rewarding the provision of this service.

- The Packet Purse Model. In this approach, the originator of the packet pays for the packet forwarding service. The service charge is distributed among the forwarding terminodes in the following way. When sending the packet, the originator loads it with a number of beans. Each forwarding terminode acquires one or several beans (depending on the direct connection, on which the packet is forwarded) from the packet and, thus, increases the stock of its beans. If a packet does not have enough beans to be forwarded, then it is discarded.

- The Free Trade of Packets Model. In this approach, the destination of the packet pays for the packet forwarding service. Instead of loading the packet with beans, the originator sells it to an intermediate terminode on the route to the destination for some beans.

\footnotetext{
${ }^{4}$ Note that in military networks self-organization is not required at this level. Indeed, these networks can rely on a hierarchically organized system of certification authorities, which are represented by headquarters at different levels. Self-organization of the network is required only in the battlefield, which does not effect the key management problem in such a radical way as it does in the Terminode network.
} 
The intermediary, then, tries to sell it to the next intermediary or to the destination for more beans. This model works if we assume that the price of the packet increases as the packet gets closer to its destination. This seems to be reasonable given that the closer the destination is, the smaller the risk that the packet cannot be delivered is, and thus the higher the demand for the packet is.

Cryptographic means to implement beans are under study, assuming that the terminodes have some tamper resistant hardware that can be used for bean management. The basic problems to be solved are related to bean forgery, protection of the packet purse integrity (in the Packet Purse Model), and fair exchange of packets for beans (in the Free Trade of Packets Model). The challenge is to find a trade-off between the robustness of the solution and its efficiency; forwarding a single packet should not require complex cryptographic protocols and heavy computational effort, because the cost of these may well exceed the value of the service.

\section{Application Scenarios and Discussion}

Increasingly often, replacing a technology can be more cost-effective than enhancing it. The newer the technology, the richer are the potentials for new services.

Infrastructure-less mobile networks can be an appropriate solution in a number of situations. A first example is a natural disaster. An earthquake, a hurricane, or a flood can severely damage the wired and wireless infrastructure of a region. At the same time, the need to communicate increases dramatically in order to organize relief and assistance. Terminodes can be a way to keep communications operational: even if some of them are lost or destroyed in the disaster, the remaining ones will spontaneously organize themselves to support the traffic.

A second example is related to political instability. Too often, because of a high level of corruption or because of guerilla activities, the communication network of a given region or country does not have the appropriate level of dependability. Such a situation can significantly hamper development and progress toward democracy. By empowering citizens with the networking functions, the terminodes can be an efficient solution to this kind of problem.

Terminodes can also be used for a wide range of applications in situations less critical than the ones described earlier. As mentioned in section 2, they can serve to support a kind of "citizen band", by which people could avoid having to go through the infrastructure of a given operator - due to cost or privacy concerns.

\section{Conclusion}

In this paper, we have stated the main objectives of our work and we have sketched the solutions we are exploring. Currently we are working in the following directions. First, we are refining the requirements, notably in terms of scalability, with prominent humanitarian organizations. Second, we are checking the robustness of our solutions, notably by means of simulations. Third, we are building analytical models for the most involved parts of the design, such as the radio architecture and the packet forwarding principle.

The long-term goal is to design a device that will empower citizens with direct communication facilities. 


\section{Acknowledgements}

We would like to thank Christian Bonnet, Thomas Gross, Martin Vetterli, and all the team of the Terminodes project for interesting and useful discussions.

\section{References}

[802.11] ISO/IEC 8802-11:1999(E) ANSI/IEEE Std 802.11. Part 11: Wireless LAN Medium Access Control (MAC) and Physical Layer (PHY) specifications. 1 edition, 1999 .

[BCS99] S. Basagni, I. Chlamtac, and V.R. Syrotiuk. Geographic Messagging in Wireless Ad Hoc Networks. VTC99 Huston, 1999.

[BCSW99] S. Basagni, I. Chlamtac, V.R. Syrotiuk, and B.A. Woodward. A Distance Routing Effect Algorithm for Mobility (DREAM). MOBICOM'99 Seattle, 1999.

[Blu99] Bluetooth. Specification of the Bluetooth System, v.1.0 A Core. 1 edition, 1999. available from http://www.bluetooth.com.

[For94] W. Ford. Computer Communications Security - Principles, Standard Protocols and Techniques. Prentice Hall, Inc., 1994.

[Gir91] M. Girault. Self-certified Public Keys. In Advances in Cryptology - EUROCRYPT'91, pages 490-497. Springer-Verlag, 1991.

[GH00] S. Giordano and M. Hamdi. Technical Report, EPFL-ICA, in preparation, Feb, 2000 .

[GLAF99] J. J. Garcia-Luna-Aceves and Chane L. Fullmer. Floor Acquisition Multiple Access (FAMA) in Single-channel Wireless Networks. In Mobile Networks and Applications, volume 4, pages 157-174, 1999.

[HLGH99] J.-P. Hubaux, J.-Y. Le Boudec, S. Giordano, and M. Hamdi. The Terminode Project: Toward Mobile Ad-Hoc WANs. In Proc. of Workshop on Mobile, Multimedia Conference, San-Diego, 1999.

[HP99] Z.J. Haas and M.R. Pearlman. Determining the Optimal Configuration for the Zone Routing Protocol. IEEE JSAC, August 1999.

[Hu93] Limin Hu. Distributed Code Asignments for CDMA Packet Radio Networks. IEEE/ACM Transactions on Networking, 1(6):668-677, 1993.

[Hub99] J.-P. Hubaux. Terminodes : Toward Self-organized Mobile Networks. Technical Report SSC/1999/022, EPFL-ICA, June, 1999.

$\left[\mathrm{ICP}^{+} 99\right] \quad$ A. Iwata, C.-C. Chiang, G. Pei, M. Gerla, and T.-W. Chen. Scalable Routing Strategies for Ad-hoc Wireless Networks. IEEE JSAC, 1999.

[JT87] J. Jubin and J.D. Tornow. The DARPA Packet Radio project. Proceedings of the IEEE., 1987. 
[Kel99] Frank Kelly. Mathematical modelling of the Internet . In Proc. of Fourth International Congress on Industrial and Applied Mathematics, 1999.

[KV98] Youngbae Ko and N. H. Vaidya. Location-Aided Routing (LAR) Mobile Ad Hoc Networks. MOBICOM'98 Dallas, 1998.

[MBJJ99] David A. Maltz, Josh Broch, Jorjeta Jetcheva, and David B. Johnson. The Effects of On-Demand Behavior in Routing Protocols for Multi-Hop Wireless Ad Hoc Networks. IEEE JSAC, August 1999.

[MAN] Mobile Ad-hoc Networks (MANET) WG. Mobile Ad-hoc Networks (MANET) Charter. Wg charter, IETF, 1999. http://www.ietf.org/html.charters/manetcharter.html.

[MZ99] A.B. MCDonald and T.F. Znati. A Mobility-Based Framework for Adaptive Clustering in Wireless Ad Hoc Networks. IEEE JSAC, August 1999.

[OP98] Tero Ojanpera and Ramjee Prasad. An Overview of Air Interface Multiple Access for IMT-2000/UMTS. IEEE Communications Magazine, 36(9):82-95, 1998.

[SA99] F. Stajano and R. Anderson. The Resurrecting Duckling: Security Issues for Ad-hoc Wireless networks. In Proceedings of the 7th International Workshop on Security Protocols, Cambridge, UK, April 1999.

[Sha85] A. Shamir. Identity-based Cryptosystems and Signature Schemes. In Advances in Cryptology - CRYPTO'84, pages 47-53. Springer-Verlag, 1985.

[She96] Timothy J. Shepard. A Channel Access Scheme for Large Dense Packet Radio Networks. In Proc. of SIGCOMM'96, pages 219-230, CA, USA, August 1996.

[TK75] F. Tobagi and L. Kleinrock. Packet Switching in Radio Channels: Part II - the Hidden Terminal Problem and the Busy-tone Solution. IEEE Transactions on Communications, 23(12):1417-1433, 1975.

[TN] www.terminodes.org, 1999.

[UMT97] Universal Mobile Telecommunications System (UMTS). Technical Report TR 101 146, ETSI - Eureopean Telecommunications Standards Institute, F-06921 Sophia Antipolis Cedex - FRANCE, December 1997. available from http://www.etsi.org.

[Ver98] S. Verdu. Multiuser Detection. Cambridge university, 1998.

[WESW98] Hagen Woesner, Jean-Pierre Ebert, Morten Schlager, and Adam Wolisz. Powersaving Mechanisms in Emerging Standards for Wireless LANs: The MAC Level Perspecitve. IEEE Personal Communications, pages 40-48, June 1998.

[ZH99] L. Zhou and Z. Haas. Securing Ad Hoc Networks. IEEE Network, 13(6):24-30, November/December 1999.

[Zim95] P. Zimmermann. The Official PGP User's Guide. MIT Press, 1995. 